\title{
An Enskog Correction for Size and Mass Difference Effects in Mixture Viscosity Prediction*
}

\author{
James F. Ely ${ }^{\dagger}$ \\ National Bureau of Standards, Boulder, CO 80303
}

\begin{abstract}
May 26, 1981
A method is presented which corrects the one-fluid conformal solution viscosity model for size and mass difference effects. This correction, which is based on the Enskog model for hard sphere mixtures, is empirical as applied to transport but has a rigorous basis in equilibrium theory. Comparisons of predictions and experimental viscosities for 24 binary mixtures are presented.
\end{abstract}

Key words: Corresponding states; Enskog theory; mass difference effects; mixture viscosity; size difference effects.

\section{Introduction}

In a series of recent reports [1, 2, 3], Ely and Hanley have proposed a corresponding states method for the prediction of the viscosity and thermal conductivity of pure hydrocarbons and their mixtures. This work was an extension of the previous work of Hanley $[4,5]$, which dealt with the transport properties of liquefied natural gas mixtures, to molecular weight ranges corresponding to $\mathrm{C}_{20}$ and other chemical types (e.g., aromatics). The method is based on a one-fluid, conformal solution concept and requires only pure component, equilibrium parameters such as the critical parameters as input. No transport data are required.

Extensive comparisons of the predictions of the model with experimental data have been reported and are summarized in tables 1 and 2 . In general the results are excellent with the average absolute error between experiment and prediction being less than 8 percent for both pure fluids and mixtures. It was noted, however, that when the size difference of two binary mixture species becomes large (e.g., $V_{1}^{c} / V_{2}^{c} \sim 6$ ), the predictions of the viscosity model become markedly worse. This failure of the one-fluid model for viscosity has been explained by the nonequilibrium molecular dynamics studies of Hanley and Evans [6, 7]. These studies have shown that for mixtures of molecules of substantially different size, the mean density approximation inherent in the one-fluid theory for the binary pair distribution function fails, even for a conformal mixture. A conse-

\footnotetext{
*Partially supported by the U.S. Department of Energy, Office of Basic Energy Sciences, Contract No. DE-A101-76PR06010.

†Thermophysical Properties Division, National Engineering Laboratory.

${ }^{1}$ Raised figures indicate literature references located at the end of this paper.
}

quence of this failure is that the local or ambient concentration of the mixture components is not the same as the bulk concentration. This is shown in figure 1 for a 50/50 mixture of a conformal system whose size difference is two [7]. As one can see, the concentration of the larger component about a central large molecule $\left(x_{22}\right)$ is greater than the bulk

TABLE 1. Summary of One-Fluid Corresponding States Viscosity Results for Pure Fluids. ${ }^{a}$

\begin{tabular}{lrrr}
\hline \hline \multicolumn{1}{c}{ Fluid Type } & N & AAD & \multicolumn{1}{c}{ BIAS } \\
\hline n-Paraffins & 1301 & 4.89 & -0.48 \\
i-Paraffins & 155 & 21.17 & -21.17 \\
Alkenes & 58 & 11.29 & 7.85 \\
Cycloalkanes & 89 & 40.56 & -40.56 \\
Alkylbenzenes & 155 & 8.45 & -0.69 \\
Carbon Dioxide & 111 & 4.75 & -4.53 \\
\hline Overall & 1869 & 8.42 & -4.10 \\
\hline
\end{tabular}

- AAD $=$ Average absolute percent deviation. BIAS = Average percent deviation.

TABle 2. Summary of One-Fluid Corresponding States Viscosity Results for Binary Mixtures."

\begin{tabular}{lrrr}
\hline Mixture Type & $\mathrm{N}$ & AAD & BIAS \\
\hline Alkane/Alkane & 303 & 5.89 & -1.79 \\
Alkane/Cycloalkane & 24 & 17.31 & -16.51 \\
Alkane/Alkylbenzene & 128 & 7.41 & -0.01 \\
\hline Overall & 455 & 6.95 & -2.07 \\
\hline
\end{tabular}

- AAD = Average absolute percent deviation. BIAS = Average percent deviation.

These results were obtained using the empirical size difference correction proposed by Ely and Hanley [1]. 


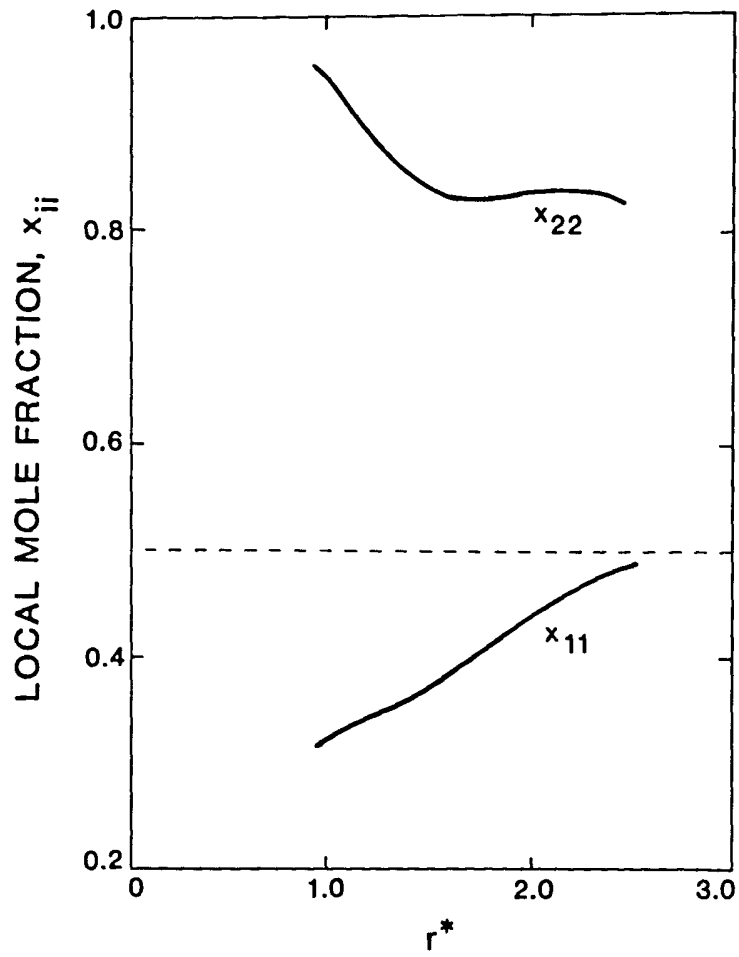

FICURE 1. Local mole fractions for a conformal mixture of soft spheres with a mass ratio of 10 and size ratio of 2 plotted versus reduced intermolecular separation $r^{*}[7]$.

concentration until one exceeds four or five molecular diameters. Since the viscosity (as well as other thermophysical properties) are determined from relatively short-range forces, the large component dominates the value of the mixture viscosity. Ely and Hanley [1] attempted to correct for this effect with an empirical relation based on the size ratios in the mixture. Although this function was somewhat successful (as is reflected in table 2), the size difference effects persist in the model predictions.

In this manuscript, a systematic correction to the onefluid viscosity model is proposed for size and mass difference effects. This correction is based on the exact solution of the Enskog model for a multicomponent mixture of hard spheres [8]. This approach has a rigorous foundation in the perturbation expansion of an equilibrium property of a fluid [9], but is empirical as applied to transport phenomena. In spite of this empiricism, the proposed correction does improve the viscosity predictions for mixtures which exhibit large size and mass differences, for both the dense liquid and dilute gas states.

Section 2 of this article summarizes the assumptions and working equations of the one-fluid, conformal solution viscosity model (CSVM). Section 3 discusses the hard sphere expansion model and describes the Enskog solution for a multicomponent mixture of hard spheres which is the ana. lytical formulation used to correct the CSVM. In section 4 the predictions of the corrected and uncorrected models are compared with experimental data for both the dilute gas and high density fluids. Unfortunately, for methane/ndecane like systems where the size and mass difference effects would be the most pronounced, no dilute gas experimental viscosities have been measured. For this reason, the model predictions are also compared to calculated Lennard. Jones viscosities.

\section{One-Fluid Viscosity Model}

In the one-fluid conformal solution viscosity model there are three basic assumptions: (1) the viscosity $(\eta)$ of a mixture at a density $\varrho$, temperature $T$ and composition $\left\{x_{\alpha}\right\}$ can be equated to the viscosity of a hypothetical pure fluid, i.e., $\eta_{\operatorname{mix}}\left(\varrho, T,\left\{x_{\alpha}\right\}\right)=\eta_{x}(\varrho, T) ;(2)$ the viscosity of the hypothetical pure fluid may be evaluated via a corresponding states principle

$$
\eta_{x}(\varrho, T)=\eta_{0}\left(\varrho_{o}, T_{0}\right) F_{\eta}
$$

where $F_{\eta}$ is a dimensional factor defined below and (3) the reference fluid density and temperature $\left(\varrho_{o}\right.$ and $\left.T_{o}\right)$ may be evaluated via an extended equilibrium corresponding states principle [10] viz.

$$
\varrho_{o}=\varrho h_{x} \text { and } T_{o}=T / f_{x}
$$

where $h_{x}$ and $f_{x}$ are defined by the relations

$$
A_{x}^{R}(\varrho, T)=f_{x} A_{o}^{R}\left(\varrho h_{x} T / f_{x}\right)
$$

and

$$
Z_{x}(\varrho, T)=Z_{o}\left(\varrho h_{x}, T / f_{x}\right)
$$

$A^{R}$ denotes the residual Helmholtz free energy of the hypothetical or reference fluid (subscripts $x$ or $o$, respectively) and $Z$ is the compressibility factor, $p / \varrho R T$. In eq (1), $F_{\eta}$ is given by

$$
F_{\eta}=\left(\frac{M_{x}}{M_{o}}\right)^{1 / 2} f_{x}^{1 / 2} h_{x}^{-2 / 3}
$$

where $M$ denotes the mass.

In order to apply the model to pure fluids or mixtures, analytical expressions for $f_{x}, h_{x}$, and $M_{x}$ as well as for the reference fluid equation of state and viscosity surface are required. In our previous work, methane was chosen as the reference fluid owing to the availability of $p V T$ and viscosity data for that fluid. The appropriate correlations have been reported previously [1] and will not be repeated here. 
For a mixture, $f_{x}, h_{x}$, and $M_{x}$ must be obtained via mixing and combining rules for the corresponding mixture component parameters. We have adopted a set of one-fluid mixing rules given by the following

$$
\begin{gathered}
f_{x}=h_{x}^{-1} \sum_{\alpha} \sum_{\beta} x_{\alpha} x_{\beta} f_{\alpha \beta} h_{\alpha \beta} \\
h_{x}=\sum_{\alpha} \sum_{\beta} x_{\alpha} x_{\beta} h_{\alpha \beta}
\end{gathered}
$$

and

$$
M_{x}=\left[\sum_{\alpha} \sum_{\beta} x_{\alpha} x_{\beta} m_{\alpha \beta}^{1 / 2} f_{\alpha \beta}^{1 / 2} h_{\alpha \beta}^{4 / 3}\right]^{2} f_{x}^{-1} h_{x}^{-8 / 3}
$$

The combining rules for the binary pair parameters (as denoted by an " $\alpha \beta$ " subscript) are given by

$$
\begin{gathered}
f_{\alpha \beta}=\left(f_{\alpha} f_{\beta}\right)^{1 / 2}\left(1-k_{\alpha \beta}\right) \\
h_{\alpha \beta}=\frac{1}{8}\left(h_{\alpha}^{1 / 3}+h_{\beta}^{1 / 3}\right)^{3}\left(1-l_{\alpha \beta}\right)
\end{gathered}
$$

and

$$
m_{\alpha \beta}=2 m_{\alpha} m_{\beta}\left[m_{\beta}+m_{\beta}\right] .
$$

In eqs (7) and (8) $k_{\alpha \beta}$ and $l_{\alpha \beta}$ are the binary interaction parameters which can be set equal to zero in viscosity predictions. The parameters $f_{\alpha}$ and $h_{\beta}$ are the equivalent substance reducing ratios for the energy and volume for component $\alpha$ in the mixture. They are given by

$$
\begin{aligned}
& f_{\alpha}=\left(T_{\alpha}^{c} / T_{o}^{c}\right) \theta\left(V_{\alpha}^{*}, T_{\alpha}^{*}, \omega_{\alpha}\right) \\
& h_{\alpha}=\left(V_{\alpha}^{c} / V_{o}^{c}\right) \phi\left(V_{\alpha}^{*}, T_{\alpha}^{*}, \omega_{\alpha}\right)
\end{aligned}
$$

where the subscript " $c$ " indicates a critical value, "*" denotes a value reduced by the critical point and $\omega$ is Pitzer's acentric factor. $\theta$ and $\phi$ are the shape factors of Leach and Leland $[11,12]$ whose detailed functional forms are given in reference [1]. $T$ denotes the absolute temperature, $V$ is the molar volume and $m$ is the mass.

The mass mixing rule given by eq (6) was derived by Evans and Hanley [6] in their study of the viscosity of a mixture of conformal soft spheres. It arises by examining the potential contribution to the pressure tensor in terms of the nonequilibrium radial distribution function and thus is a mixing rule for the potential or in practice, high density contribution to the viscosity. This rule was adopted for all densities, however, since the emphasis of our previous work was on the dense fluid states. One might expect, therefore, that the CSVM might be somewhat less accurate for the dilute gas, kinetic regime where the mixture mass dependence is effectively proportional to $m^{1 / 2} f^{1 / 2} h^{-2 / 3}$ rather than $m^{1 / 2} f^{1 / 2} h^{4 / 3}$ as given in eq (6). This point will be discussed further in section 4 .

\section{The Enskog Correction}

Mansoori and Leland and their co-workers [9, 13] have proposed a conformal solution model for equilibrium thermodynamic properties in which a dimensionless or reduced property of a mixture is expanded about the corresponding property of a hard sphere mixture. For example, if $X_{\text {mix }}$ represents the value of the real mixture property, one obtains

$X_{m i x}\left(\varrho, T,\left\{x_{\alpha}\right\}\right)=X_{m i x}^{H S}\left(\left\{\varrho \sigma_{\alpha}^{3}\right\},\left\{x_{\alpha}\right\}\right)-X_{x}^{H S}\left(\varrho \sigma_{x}^{3}\right)+X_{o}\left(\varrho_{o}^{\prime}, T_{o}\right)$

In this equation $X_{\text {mix }}^{H S}$ denotes the value of the property in a mixture of hard spheres of diameters $\left\{\sigma_{\alpha}\right\},\left\{x_{\alpha}\right\}$ denotes the mixture composition, $X_{x}^{H S}$ is the property value in a pure hard sphere fluid of effective diameter $\sigma_{x}$ (e.g., a one-fluid approximation) and $X_{o}$ is the value obtained from a real, pure fluid reference substance, evaluated at the state point $\left(\varrho_{o}, T_{o}\right)$ where $\varrho_{o}^{\prime}=\varrho \sigma_{x}^{3} / \sigma_{o}^{3}$ and $T_{o}^{\prime}=T \epsilon_{o} / \epsilon_{x}$. In terms of intermolecular potentials the parameters $\sigma$ and $\epsilon$ correspond to the points where $u(\sigma)=0$ and $\min (u)=-\epsilon$. Both $\sigma_{x}$ and $\epsilon_{x}$ are one-fluid parameters which must be evaluated via mixing rules. The difficulty in applying this approach lies in assigning values to $\sigma$ and $\epsilon$ for the mixture components. One possible approach is to assume that $\sigma^{3} \sim V_{c}$ and $\epsilon \sim$ $T_{c}$ which leads to factors such as those given in eqs (10-11). The choice of parameters used in this work will be discussed in section 4.

Although transport properties cannot, in general, be expanded in a perturbation series [14], it is tempting to apply the hard sphere expansion (HSE) formalism to the conformal solution viscosity model presented in section 2 . Formally this may be written as

$$
\begin{gathered}
\eta_{\text {mix }}\left(\varrho, T,\left\{x_{a}\right\},\left\{m_{a}\right\}\right)=\eta_{\text {mix }}^{H S}\left(\left\{\varrho \sigma_{a}^{3}\right\},\left\{x_{a}\right\},\left\{m_{a}\right\}\right)- \\
\eta_{x}^{H S}\left(\varrho \sigma_{x}^{3}, m_{x}\right)+\eta_{o}\left(\varrho_{o}, T_{o}\right) F_{\eta}
\end{gathered}
$$

where the notation is the same as defined previously. Note that in the case of transport we must also consider the masses of the particles $\left\{m_{a}\right\}$.

In practice, we do not have an exact model for the viscosity of a hard sphere fluid (pure or mixed) at all densities. For this reason, the Enskog model [15], which has been solved for a multicomponent mixture of hard spheres by Tham and Gubbins [8], was selected to calculate $\eta_{m i x}^{H S}$ and $\eta_{x}^{H S}$. Their solution is given by

$$
\eta_{\text {mix }}^{E N S K O G}=\sum_{i} \beta_{i}(T, \varrho) Y_{i}+\frac{48}{15 \pi} \sum_{i} \sum_{j} x_{i} x_{j} u_{i j}^{o} y_{i j}
$$


where

$$
Y_{i}=x_{i}\left[1+4 / 5 \sum_{j} x_{j} M_{j i} y_{i j}\right]
$$

and the $\beta_{i}$ are the solutions to the set of linear equations defined by the following

$$
\sum_{j} B_{i j} \beta_{j}=Y_{i}
$$

where

$$
B_{i j}=2 \sum_{\ell} x_{i} x_{\ell} y_{i l} M_{\ell i}^{2}\left(u_{i l}^{\circ}\right)^{-1}\left[\left(1+5 / 3 \frac{m_{i}}{m_{\ell}}\right) \delta_{i j}-2 / 3\left(\frac{m_{i}}{m_{\ell}}\right) \delta_{j \ell}\right] .
$$

In these equations, $b_{i j}=2 \pi \sigma_{i j}^{3} / 3, \sigma_{i j}=1 / 2\left(\sigma_{i}+\sigma_{j}\right), M_{j l}=$ $m_{j} /\left(m_{l}+m_{j}\right), u_{i j}^{\circ}=b_{i j} \mathrm{n} \eta_{i j}^{\circ}, \eta_{i j}^{\circ}=5 / 16\left(\frac{m_{i j} k T}{\pi}\right)^{1 / 2} \sigma_{i j}^{-2}, m_{i j}=$ $2 m_{i} M_{j i}, y_{j}=n b_{i j} g_{i j}\left(\sigma_{i j}\right), n=$ number density, $g_{i j}$ is the hard sphere radial distribution function for the $i j$ pair, and $\delta$ is the Kronecker delta function. Although these equations are somewhat complex, they may be readily solved on a digital computer.

The final model, which we shall call the hard sphere expansion-conformal solution viscosity model (HSE-CSVM), is given by

$\eta_{\text {mix }}\left(\varrho, T,\left\{x_{a}\right\},\left\{m_{a}\right\}\right)=\Delta \eta^{\text {ENSKOG }}+\eta_{o}\left(\varrho_{o}, T_{o}\right) F_{\eta}$

with

$$
\begin{aligned}
\Delta \eta^{\text {ENSKOG }}= & \eta_{m i x}^{\text {ENSKOG }}\left(\left\{\varrho \sigma_{a}^{3}\right\},\left\{x_{a}\right\},\left\{m_{a}\right\}\right)- \\
& \eta_{x}^{\text {ENSKOG }}\left(\varrho \sigma_{x}^{3}, m_{x}\right)
\end{aligned}
$$

\section{Results}

In order to apply eqs (14) and (15) values for the hard sphere diameters $\sigma_{a}$ must be chosen and mixing rules for the one-fluid values $\sigma_{x}$ and $m_{x}$ in the hard sphere system must be selected. As was mentioned previously, consid. erable freedom exists for the choice of the molecular diameters. Unfortunately, the hard sphere contributions to the viscosity are rather sensitive to this choice, thus several possibilities were considered. The relationship which was selected is given by

$$
\sigma_{a}=\left(V_{o}^{c} h_{a} / 3.058 N_{o}\right)^{1 / 3}
$$

where $h_{a}$ is the ratio defined in eq (11) and is obtained in the CSVM calculations and $N_{0}$ is Avogadro's number. The factor 3.058 was chosen so that methane would have a diameter of $3.75810^{-10} \mathrm{~m}$ which corresponds to the Lennard-Jones
(12-6) intermolecular potential value given by Reid, et al. [16]. This choice, although resonable, is still somewhat arbitrary. The mixing rules for the one-fluid hard sphere system were chosen to be consistent with those used in the CVSM, eqs (5) and (6), viz.

$$
\sigma_{x}^{3}=\sum_{a} \sum_{b} x_{a} x_{b} \sigma_{a b}^{3}
$$

and

$$
M_{x}=\left[\sum_{a} \sum_{b} x_{a} x_{b} m_{a b}^{1 / 5} \sigma_{a b}^{4}\right]^{2} \sigma_{x}^{-8}
$$

where $\sigma_{a b}=1 / 2\left(\sigma_{a}+\sigma_{b}\right)$ and $m_{a b}$ is defined in eq (9).

\subsection{Results for the Dilute Gas Limit}

The correction was first tested on the dilute gas viscosity of a methane/propane mixture with the results being given in table 3. This table gives the experimental data [17], CSVM predictions and the HSE-CSVM predictions obtained using eqs (14-16). As one can see, the HSE-CSVM is consistently more accurate with average absolute percentage deviation being 3.6 percent as compared to 6.29 percent for the CSVM.

The size and mass difference in the methane/propane system is not very great $-\sigma_{1} / \sigma_{2} \sim 1.4$ and $m_{1} / m_{2} \sim 3$. One would expect that the effect of the correction would be more pronounced in a system like methane/n-decane where the size and mass ratios are 1.8 and 9 , respectively. Unfortunately no experimental measurements for the dilute gas viscosity of this mixture have been reported. For this reason, the Lennard-Jones gas viscosities of this system were calculated using the standard kinetic theory formalism [15]. Although it is impossible to assess the absolute accuracy of these values, they do serve as a rational basis upon which the HSE-CSVM and CSVM may be compared.

In order to perform the dilute gas Lennard-Jones (LJ) calculations, values for the intermolecular potential minimum $\epsilon_{a}$ for the mixture components must be chosen as well as values for the $\sigma_{a}$. The $\sigma_{a}$ were obtained from eq (18) and $\epsilon_{a} / k$ where $k$ is Boltzmann's constant were calculated from the empirical relation

$$
\epsilon_{a} / k=T_{o}^{c} f_{a} / 1.282
$$

where $f_{a}$ is the CSVM reducing parameter given in eq (10). The factor 1.282 was chosen so that methane would have a value of $\epsilon / k$ of 148.6 which is the Lennard-Jones value reported by Reid, et al. [16].

Table 4 summarizes the results obtained with the $\mathrm{LJ}$ kinetic theory model and the HSE-CSVM and CSVM at three compositions and four temperatures. Comparing the 
TABLE 3. Comparison of Calculated and Experimental [17] Dilute Gas Viscosities of Methane/Propane Mixtures at I atm.

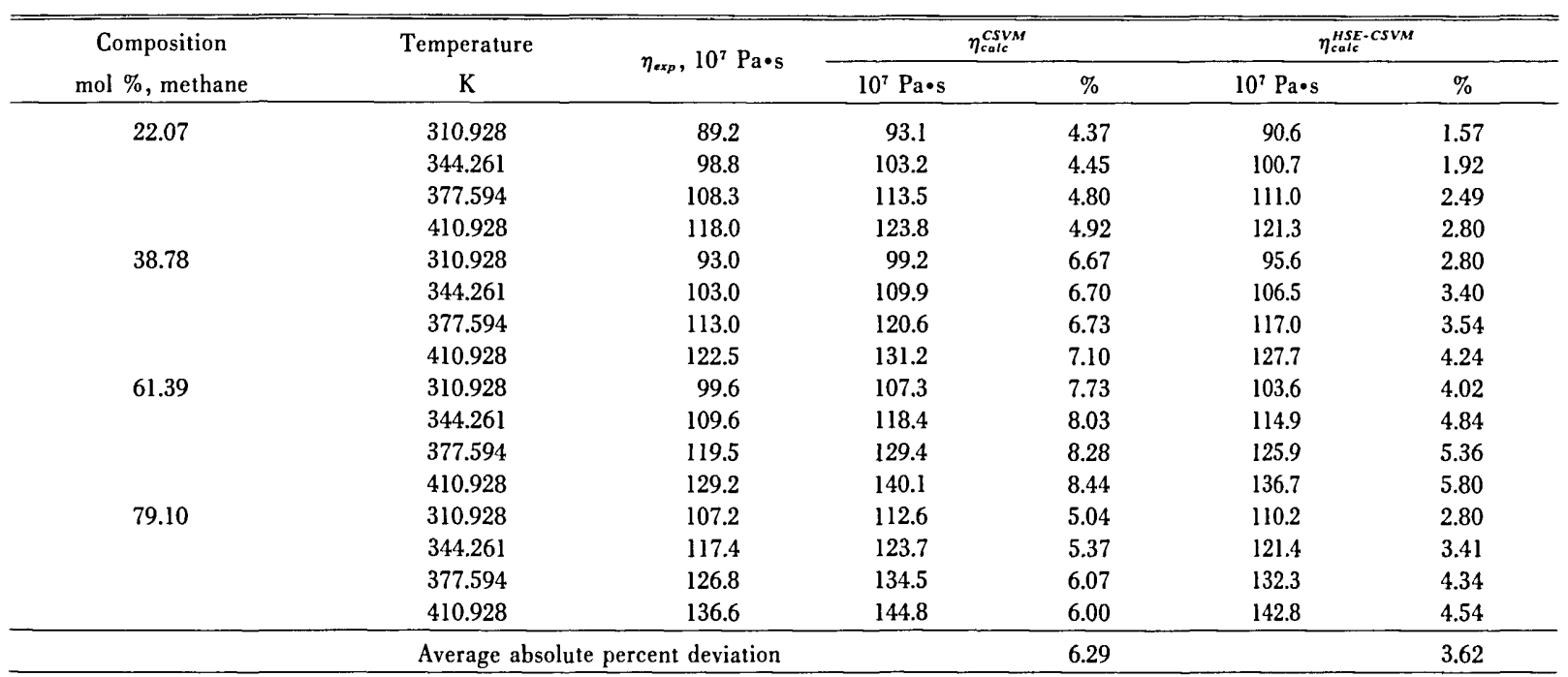

TABLE 4. Comparison of Calculated and Lennard-Jones (12-6) Dilute Gas Viscosities of Methane/n.Decane Mixtures and CSVM Predictions

\begin{tabular}{|c|c|c|c|c|c|c|}
\hline \multirow{2}{*}{$\begin{array}{c}\text { Composition } \\
\text { mol \%, methane }\end{array}$} & \multirow{2}{*}{$\begin{array}{c}\text { Temperature } \\
\mathrm{K} \\
\end{array}$} & \multirow{2}{*}{$\eta_{e x p}^{L}, 10^{7} \mathrm{~Pa} \cdot \mathrm{s}$} & \multicolumn{2}{|c|}{$\eta_{\text {caic }}^{\text {cSVM }}$} & \multicolumn{2}{|c|}{$\eta_{\text {calc }}^{H S E-C S V M}$} \\
\hline & & & $10^{7} \mathrm{~Pa} \cdot \mathrm{s}$ & $\%$ & $10^{7} \mathrm{~Pa} \cdot \mathrm{s}$ & $\%$ \\
\hline \multirow[t]{4}{*}{25.0} & 300 & 46.5 & 54.9 & 18.06 & 43.4 & -6.67 \\
\hline & 400 & 65.4 & 76.2 & 16.51 & 62.8 & -3.98 \\
\hline & 500 & 86.4 & 99.2 & 14.81 & 84.1 & -2.66 \\
\hline & 600 & 108.8 & 124.2 & 14.15 & 107.6 & -1.10 \\
\hline \multirow[t]{4}{*}{50.0} & 300 & 56.4 & 74.9 & 32.80 & 50.1 & -11.17 \\
\hline & 400 & 79.2 & 103.1 & 30.18 & 74.9 & -5.43 \\
\hline & 500 & 103.7 & 133.4 & 28.64 & 102.4 & -1.25 \\
\hline & 600 & 129.1 & 165.2 & 27.96 & 132.3 & 2.48 \\
\hline \multirow[t]{6}{*}{75.0} & 300 & 75.2 & 104.2 & 38.56 & 70.6 & -6.12 \\
\hline & 400 & 103.6 & 140.5 & 35.62 & 104.8 & 1.16 \\
\hline & 500 & 132.2 & 177.0 & 33.89 & 140.5 & 6.28 \\
\hline & 600 & 160.7 & 211.5 & 31.61 & 175.4 & 9.15 \\
\hline & Average a & percent deviation & & 20.17 & & 3.59 \\
\hline & Average $p$ & eviation & & 20.17 & & -2.40 \\
\hline
\end{tabular}

results we see a substantial difference in the CSVM predictions and the $\mathrm{LJ}$ calculations. In the case where the methane content is high, (75 percent) the HSE-CSVM is in better agreement with the $\mathrm{LJ}$ calculations by $20-30$ percent. Also, the overall absolute percentage deviation for the HSECSVM is 3.6 percent as opposed to 20 percent for the CSVM version. Although the accuracy of the $\mathrm{LJ}$ calculations is uncertain, similar calculations on the methane/propane system where experimental data do exist yielded agreement between the $\mathrm{LJ}$ and experiment of better than 2 percent. One can postulate, therefore, that discrepancies similar to those shown in table 4 would exist between the CSVM and real experimental data for the methane/n-decane system.

Note that in both comparisons the CSVM predicts values which are too large in the dilute gas limit. We attribute this to the potential or high density mass mixing rule which is used in $F_{\eta}$ in eq (3). Thus, we see that in the low density limit the HSE Enskog correction is negative and lowers the predicted viscosity.

\subsection{High Densiły Results}

The initial motivation for this work was to obtain a correction for the effect of size differences on the predicted high density viscosity of systems like methane/n-decane mixtures. Figure 2 compares the experimental [18] and CSVM predicted viscosities of methane/n-decane mixtures as a function of reduced density at three different compositions. Note that the predictions are worst for the high methane 


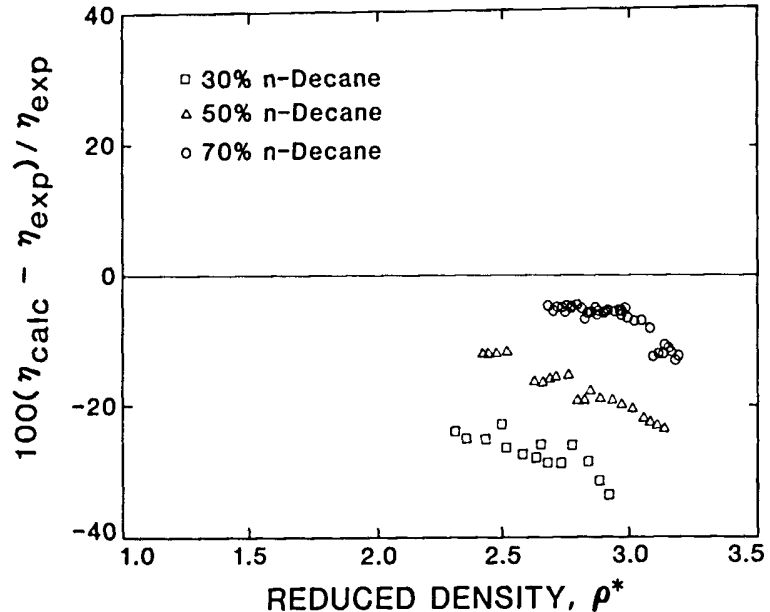

FICURE 2. Comparison of calculated and experimental viscosities of methane/n-decane mixtures using the uncorrected model. Note that the predictions are worst ( $\sim 30$ percent error) for the highest methane concentration.

composition and improve with increasing decane content. This is somewhat surprising since methane is the reference fluid in the CVSM calculations. This result was explained in the introduction and is attributable to a failure of the onefluid theory to adequately represent the size difference effects in the high density region.

Figure 3 compares the experimental and calculated results for the HSE-CSVM. We see in this case that there is a marked improvement in both the bias and overall deviation. Note that in this case the HSE correction is positive. The density dependence of the HSE correction is illustrated in figure 4 which shows $\frac{\Delta \eta}{T^{1 / 2}}=\left(\eta_{\operatorname{mix}}^{\text {ENSKOG }}-\eta_{x}^{\text {ENSKOO }}\right) / T^{1 / 2}$ plotted against reduced density at a size ratio of $\sigma_{1} / \sigma_{2}=2.0$ and mass ratio of $m_{1} / m_{2}=8$ at three compositions. This corresponds approximately to a methane/decane like system. In figure 4 the density was reduced by an approximation to the critical density of the mixture given by $\mathrm{e}_{\mathrm{c}}^{-1}=$ $3.058 N_{0}\left(x_{1} \sigma_{1}^{3}+x_{2} \sigma_{2}^{3}\right)$. This figure demonstrates that the correction decreases in magnitude with increasing concentration of the larger component $\left(x_{1}\right)$ and is small and negative below reduced densities of 1.5 . Also the correction increases sharply above reduced densities of 1.5.

Table 5 compares the overall predictions for both the corrected and uncorrected model for 24 binary systems. The literature references for the experimental data are given in [1]. The systems where the size difference is large $\left[\sigma_{2} / \sigma_{1} \approx\right.$ $\left.\left(V_{2}^{c} / V_{1}^{c}\right)^{i / 3}\right]$ show substantial improvement while those of similar size are essentially unchanged. It may be possible to further improve the corrected model by a judicious choice of the $\sigma_{a}$. This possibility is currently being examined.

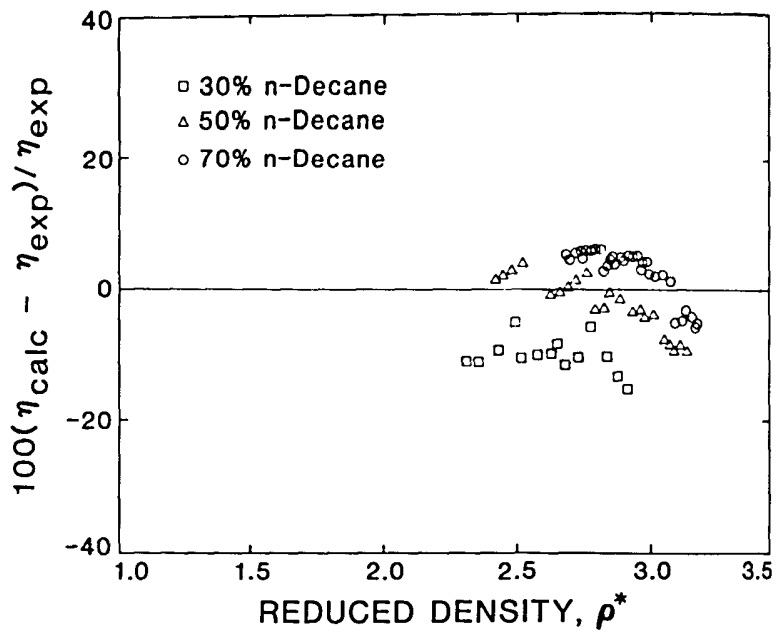

Ficure 3. Comparison of calculated and experimental viscosities of methane/n-decane mixtures using the corrected model. Note that both the bias and average absolute deviations are improved.

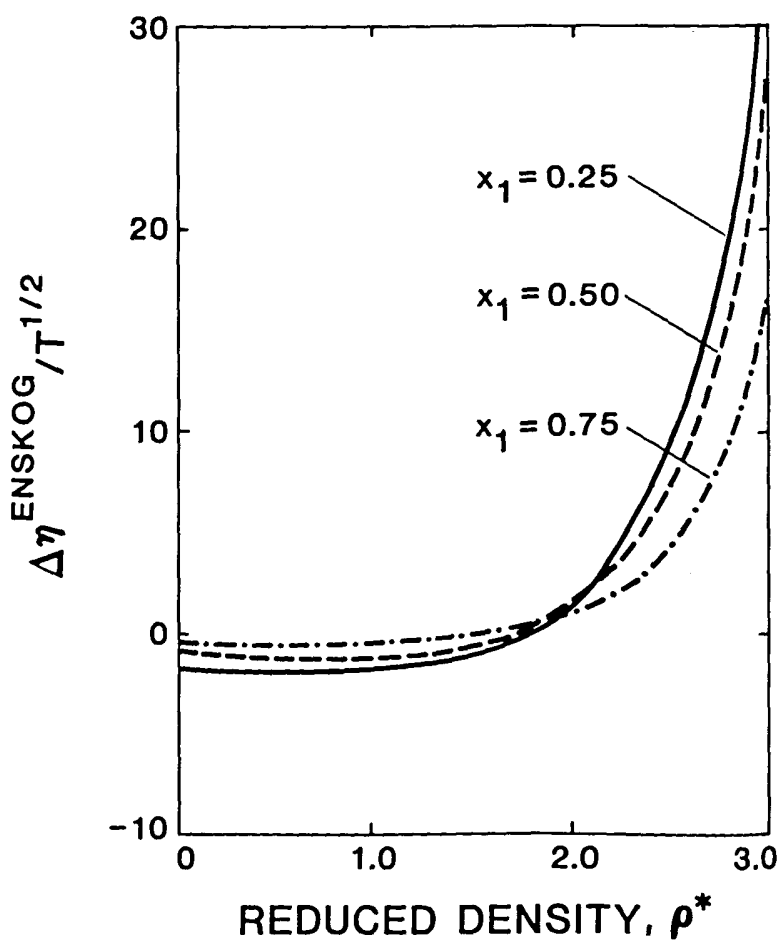

Ficure 4. Plot of $\Delta \eta^{E N S K O G} / T^{1 / 2}$ versus reduced density at three compositions of the larger component $\left(x_{1}\right), \sigma_{1} / \sigma_{2}=2$ and $m_{1} / m_{2}=8$ which roughly corresponds to a methane/n-decane system. 
TABLE 5. Summary of Calculated and Experimental Dense Fluid Binary Mixture Viscosities. ${ }^{\circ}$

\begin{tabular}{|c|c|c|c|c|c|c|c|}
\hline Component 1 & Component 2 & $\sigma_{2} / \sigma_{1}$ & $\mathrm{~N}$ & $\mathrm{AAD}^{c S V M}$ & BIAS $^{c S V M}$ & $\mathrm{AAD}^{H S E-C S V M}$ & BIAS $^{H S E \cdot C S V M}$ \\
\hline \multirow{3}{*}{ Methane } & Propane & 1.273 & 134 & 5.91 & -5.29 & 4.62 & -3.45 \\
\hline & n-Nonane & 1.778 & 32 & 6.37 & -5.58 & 4.12 & -2.61 \\
\hline & n-Decane & 1.839 & 71 & 14.43 & -14.43 & 5.35 & -1.54 \\
\hline \multirow[t]{2}{*}{ 2,3-Dimethylbutane } & $\mathrm{n} \cdot \mathrm{Hexane}$ & 1.014 & 2 & 5.32 & -5.32 & 5.31 & -5.31 \\
\hline & $\mathrm{n}-0$ ctane & 1.110 & 2 & 6.03 & -6.03 & 5.65 & -5.65 \\
\hline \multirow[t]{2}{*}{ n-Hexane } & n-Tetradecane & 1.304 & 10 & 2.15 & -1.15 & 1.92 & 0.54 \\
\hline & n-Hexadecane & 1.356 & 26 & 4.04 & -3.85 & 2.59 & -1.97 \\
\hline \multirow[t]{4}{*}{$\mathrm{n} \cdot$ Heptane } & n-Dodecane & 1.185 & 3 & 2.47 & 2.47 & 3.44 & 3.44 \\
\hline & n-Tetradecane & 1.242 & 3 & 1.19 & 0.10 & 1.82 & 1.51 \\
\hline & $\mathrm{n}$-Hexadecane & 1.291 & 3 & 3.03 & -3.03 & 2.52 & -1.32 \\
\hline & n-Octadecane & 1.333 & 2 & 2.95 & -2.95 & 1.92 & -0.71 \\
\hline $\mathrm{n} \cdot 0$ ctane & n-Decane & 1.074 & 2 & 3.09 & 3.09 & 3.27 & 3.27 \\
\hline n-Tetradecane & $\mathrm{n}$-Hexadecane & 1.040 & 11 & 2.31 & 2.08 & 2.32 & 2.10 \\
\hline \multirow[t]{8}{*}{ Benzene } & n-Hexane & 1.130 & 15 & 5.85 & -2.70 & 5.74 & -2.28 \\
\hline & $\mathrm{n}$-Heptane & 1.186 & 3 & 4.68 & 4.68 & 5.85 & 5.85 \\
\hline & 2,2,4-Trimethylpentane & 1.218 & 26 & 13.14 & -13.14 & 12.46 & -12.46 \\
\hline & n-Decane & 1.329 & 3 & 4.45 & 0.73 & 5.47 & 3.46 \\
\hline & n-Dodecane & 1.406 & 3 & 2.87 & 2.87 & 6.47 & 6.47 \\
\hline & n-Tetradecane & 1.473 & 3 & 2.96 & -1.47 & 3.63 & 2.55 \\
\hline & $\mathrm{n}$-Hexadecane & 1.531 & 3 & 3.75 & -2.80 & 3.76 & 1.46 \\
\hline & n-0ctadecane & 1.581 & 3 & 2.99 & -2.99 & 2.73 & 1.97 \\
\hline \multirow[t]{4}{*}{ Toluene } & n-Heptane & 1.110 & 21 & 5.15 & 5.15 & 5.32 & 5.32 \\
\hline & n-Octane & 1.157 & 20 & 9.03 & 9.03 & 9.50 & 9.50 \\
\hline & 2,2,4-Trimethylpentane & 1.140 & 28 & 6.61 & -4.64 & 6.62 & -4.37 \\
\hline & \multicolumn{2}{|l|}{ Overall } & 429 & 7.45 & -5.23 & 5.31 & -1.86 \\
\hline
\end{tabular}

${ }^{a}$ The CSVM results were obtained without the empirical size difference correction give in [1].

\section{Summary and Conclusions}

We have shown that a relatively simple correction to the one-fluid conformal solution viscosity model may be obtained from the Enskog hard sphere theory. This function effectively corrects for errors in the mass mixing rules at low density and also for size difference effects at high density. Even though the effective correction is not substantial for systems of similar size and mass, it does systematically correct the model predictions as is reflected in the BIAS as given in table 4 . In addition, when the size and mass difference is large, as in the methane/n-decane system, the improvement in the model predictions is substantial. Further work is in progress to extend this approach to other struc. tural features such as branching and polarity which are not adequately handled by the one-fluid corresponding states model.

The author would like to acknowledge Dr. J. M. Kincaid of NBS and Professor G. Stell and Dr. John Karkheck of the State University of New York at Stony Brook for providing the original computer program which calculates the mixture Enskog viscosity. Further, the author would like to acknowledge many helpful discussions with Dr. H. J. M. Hanley. Ms. Karen Bowie assisted substantially in the preparation of the manuscript.

\section{References}

[1] Ely, J. F.; Hanley, H. J. M. Prediction of transport properties. I. Viscosity of fluids and fluid mixtures. Ind. Eng. Chem. Fundam., 20(4): 323-332; 1981 November.

[2] Ely, J. F.; Hanley, H. J. M. Prediction of the viscosity and thermal conductivity in hydrocarbon mixtures-computer program TRAPP. Proceedings of the 60th annual GPA convention; 1981 March 23-25; San Antonio, TX.

[3] Ely, J. F.; Hanley, H. J. M. A computer program for the prediction of viscosity and thermal conductivity in hydrocarbon mixtures. Nat. Bur. Stand. (U.S.) Tech. Note 1039, 1981.

[4] Hanley, H. J. M. Prediction of the viscosity and thermal conductivity coefficients of mixtures. Cryogenics 16(11): 643-651; 1976 November.

[5] Rainwater, J. C.; Hanley, H. J. M. Prediction of the transport properties of natural gas and similar mixtures. Advances in Cryogenic Engineering, Vol. 23. K. D. Timmerhaus, ed. New York, NY: Plenum Press; 1978. 561-565.

[6] Evans, D. J.; Hanley, H. J. M. Viscosity of a mixture of soft spheres. Phys. Rev. A 20(4): 1648-1654; 1979 October 1.

[7] Hanley, H. J. M.; Evans, D. J. Behavior of a nonconformal mixture via computer simulation. Int. J. Thermophys., 2(1): 1-19; 1981 January.

[8] Tham, M. K.; Gubbins, K. E. Kinetic theory of multicomponent dense fluid mixtures of rigid spheres. J. Chem. Phys. 55(1): 268-279; 1971 July 1.

[9] Mansoori, G. A.; Leland, T. W. Statistical thermodynamics of mixtures; a new version for the theory of conformal solution. J. Chem. Soc. Faraday Trans. II 6(3): 320-344; 1972. 
[10] Rowlinson, J. S.; Watson, I. D. The prediction of the thermodynamic properties of fluids and fluid mixtures $-I$. The principle of corresponding states and its extensions. Chem. Eng. Sci. 24(8): 1565-1574; 1969 October 1.

[11] Leach, J. W.; Chappelear, P. S.; Leland, T. W. Use of molecular shape factors in vapor-liquid equilibrium calculations with the corresponding states principle. A.I.Ch.E. J. 14(4): 568-576; 1968 July 1.

[12] Leach, J. W. Molecular structure corrections for application of the theory of corresponding states to non-spherical pure fluids and mixtures. Ph.D. Thesis, Rice University, Houston, TX; 1967.

[13] Chang, J. I. C.; Hwu, F. S. S.; Leland, T. W. Effective molecular dimaters for fluid mixtures. Chapter 2 in Equations of state in engineering research, Advances in chemistry series Vol. 182, Chao, K. C.; Robinson, R. L., eds. Washington, DC: American
Chemical Society; 1979. 31-48.

[14] Kincaid, J. M.; Kayser, R. F. Kinetic parturbation theory for dilute gases. Phys. Lett. 78A(3): 215-216; 1980 August 4.

[15] Hirschfelder, J. O.; Curtiss, C. F.; Bird, R. B. Molecular theory of gases and liquids. New York, NY: John Wiley and Sons; 1954. 1217 p.

[16] Reid, R. C.; Prausnitz, J. M.; Sherwood, T. K. The properties of gases and liquids, 3rd edition. New York, NY: McGraw Hill; 1977.688 p.

[17] Giddings, J. G.; Kao, J. T.; Kobayashi, R. Development of a high-pressure capillary tube viscometer and its application of methane, propane and their mixtures in the gaseous and liquid regions. J. Chem. Phys. 45(2): 578-586; 1966 July 15.

[18] Lee, A. L.; Gonzalez, M. H.; Eakin, B. E. Viscosity of methane-decane mixtures. J. Chem. Eng. Data 11(3): 281-287; 1966 July 1. 